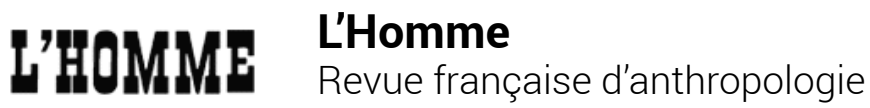

$236 \mid 2020$

Les fins de l'égalitarisme

\section{Yvette Delsaut, Carnets de socioanalyse. Écrire les pratiques ordinaires}

Cyriac Gousset

\section{OpenEdition}

1 Journals

Édition électronique

URL : https://journals.openedition.org/lhomme/38377

DOI : 10.4000//homme.38377

ISSN : 1953-8103

\section{Éditeur}

Éditions de l'EHESS

\section{Édition imprimée}

Date de publication : 16 décembre 2020

Pagination : 230-231

ISBN : 9782713228568

ISSN : 0439-4216

\section{Référence électronique}

Cyriac Gousset, "Yvette Delsaut, Carnets de socioanalyse. Écrire les pratiques ordinaires », L'Homme [En ligne], 236 | 2020, mis en ligne le 16 décembre 2020, consulté le 03 janvier 2023. URL : http:// journals.openedition.org//homme/38377 ; DOI : https://doi.org/10.4000//homme.38377 
Yvette Delsaut

Carnets de socioanalyse. Écrire les pratiques ordinaires

Éd. et préface par Andréa Daher.

Paris, Raisons d'agir, 2020, 224 p., notes bibliogr., ill. ("Cours et travaux»).

$\mathrm{L}$

A PARUTION de ces Carnets de socioanalyse est une initiative éditoriale bienvenue qui permet de réunir huit textes d'Yvette Delsaut jusqu'alors dispersés et peu accessibles, ou inédits. Cet ouvrage présente un double intérêt: celui d'être une réflexion en acte sur la place à accorder aux photographies dans l'analyse sociologique et celui de porter sur les espaces privés et les pratiques ordinaires des classes populaires. Exposer c'est, dans le cas de cette auteure, s'exposer: elle traite en effet ici du milieu populaire dont elle est originaire et révèle son intimité familiale et amicale, ce qui a été pour elle source de tourments qu'elle restitue dans un dernier chapitre réflexif. Cette «entreprise scientifique risquée» (p. 195) se retrouve au fil des pages où l'on mesure «la position inconfortable et ambiguë de l'observateur» (p. 77).

Dans ces carnets avant tout ethnographiques, Yvette Delsaut nous propose de prêter tour à tour attention aux détails d'un intérieur ouvrier («L'économie du langage populaire»), à deux cérémonies organisées lors d'un même mariage ("Le double mariage de Jean Célisse»), à l'intimité d'un vieux couple dans son logement ("L'inforjetable»), ou encore à la photo de classe de jeunes filles, élèves de troisième en 1951 ( «Une photo de classe»). Dans les deux chapitres suivants, elle rapporte le récit d'une dispute entre une jeune fille de 26 ans - qui est son amie et les propriétaires de son logement («Des excuses au masculin»), puis relate le changement d'emploi de cette dernière («Les cinq premiers jours»). Enfin, le septième carnet est consacré à un couple prenant le train ( Les Arabes du Tgv Paris-Bordeaux»). Ces objets de recherche circonscrits permettent à l'auteure de dévoiler l'étendue du caractère social de faits et gestes anodins, quotidiens, mais aussi personnels. Loin de se résoudre à un "hyper-empirisme aveugle» ou à un "théoréticisme vide» ${ }^{1}$, elle recourt à ce type d'objectivation pour définir un mode de pensée sociologique et soulever des questions méthodologiques et théoriques stimulantes. Comme le souligne l'auteure, «il s'agit à chaque fois de la mise en ouvre d'un raisonnement d'ensemble, au travers d'une illustration qui, elle, est singulière» (p. 159).

Ce raisonnement d'ensemble repose sur quelques postulats. Tout d'abord, le fait que le travail du chercheur reste nécessaire pour isoler les principes pertinents, dans la mesure où «les choses ne se donnent pas à percevoir avec la cohérence que leur confere l'exposé écrit» (p. 27). L'interprétation est donc impérative pour permettre de restituer «leur cohérence à des détails à première vue insignifiants" (p. 28). Ce qui se donne à voir n'est jamais décontextualisé des rapports sociaux: la brouille relatée dans le cinquième chapitre correspond ainsi au "protocole populaire» et respecte "la division des rôles entre les sexes et les âges" (p. 157). Dès lors, la lecture de l'ouvrage nous rappelle l'attention que l'on doit porter au social incorporé pour comprendre les pratiques. On perçoit de cette façon l'inertie ou, mieux, la durabilité des dispositions sociales incorporées lorsqu'on est plongés dans l'intimité du vieux couple du troisième carnet (pp. 88-89). Si les protagonistes en savent assez pour jouer leur rôle et sont pris au sérieux, cela ne signifie pas qu'il faudrait les considérer comme de purs êtres de raison étant donné que «la lucidité sur soi-même peut varier selon les personnes» (p. 188). Citons, enfin, les réflexions heuristiques sur la manière d'utiliser le concept d'habitus repensé par Pierre Bourdieu (pp. 186-194), qui découlent de l'observation d'un couple

1. Deux travers qu'elle avait épinglés avec Pierre Bourdieu, cf. Pierre Bourdieu \& Yvette Delsaut, "Pour une sociologie de la perception ", Actes de la recherche en sciences sociales, 1981, 40 : 9. 
dans le Tgv Paris-Bordeaux et des présupposés établis par un voyageur interprétant leurs façons d'être et de faire. Les remarques sur l'usage pertinent de la notion d'habitus sont ainsi éloignées d'une vision réductrice des déterminations sociales et "exclu[en]t qu'on puisse parler de l'habitus de quelqu'un en le qualifiant de manière lapidaire» (p. 191).

Deux textes - «Le double mariage de Jean Célisse» et "Une photo de classe» - nous semblent particulièrement intéressants en ce qu'ils témoignent du rapport de l'auteure à son milieu social d'origine qu'elle contribue à dévoiler et, plus spécifiquement, de la tension du loyalisme. Après la publication du premier texte, qui l'a "plongée dans un trouble profond et durable» (p. 206), il lui a fallu attendre dix années avant de pouvoir recommencer à «travailler sur le même milieu, avec un texte de socioanalyse basé sur du matériel autobiographique» (p. 208), et ce, sans éprouver aucune gêne, ni ressentir le besoin d'esquiver sa trajectoire sociale.

Dans le cadre du double mariage, les descriptions successives font apparaître «la tonalité si différente des deux cérémonies" (p. 76), alors même que les deux groupes d'invités partagent des propriétés sociales analogues. Cet écart est dû au passage d'un groupe restreint d'interconnaissances à un second groupe semi-anonyme dont les normes de sociabilité different. Le fait d'avoir des positions relativement proches dans l'espace social ne conduit donc pas nécessairement à une proximité affective, ce qui nous pousse à nous interroger sur les relations sociales et les degrés de censures afférents.
En se fondant sur une photo de classe datant de 1951, l'auteure livre une description minutieuse et attentive des pratiques et des représentations des jeunes filles avec qui elle a partagé sa classe de troisième. À partir de ces portraits, c'est la classe sociale qui est révélée lorsqu'elle dresse l' «inventaire des destinées scolaires des élèves représentées» (p. 105) et de l'ensemble des éléments, dont la position sociale, qui ont pesé sur ces destins sociaux. Restituant avec finesse «les charmes de l'indétermination" (p. 131) de ces années d'école, sans pour autant éluder les (nombreuses) contraintes liées notamment au genre, elle nous rappelle la "pesanteur des réalités» (p. 132) qui viennent battre en brèche les rêves de ces jeunes filles.

Ces carnets, au caractère "inachevé» (p. 7), traduisent tout l'apport et la rigueur de l'approche ethnographique d'Yvette Delsaut. Concernant l'habitus, elle insiste par exemple sur l'importance d'un travail de recherche exigeant, permettant la mise au jour d'agencements complexes d'éléments qui «s'associent au fil de l'expérience sociale» (p. 189), afin de ne pas se satisfaire de pronostics schématiques quant aux destins sociaux des individus. Il est légitime d'espérer que l'ouvrage intéresse au-delà de la communauté des chercheurs en sciences sociales car, comme le note Andréa Daher dans sa préface, de nombreux «objets exposés dans ces carnets sont précisément les mêmes que beaucoup d'entre nous gardons pendant toute une vie» (p. 6).

Cyriac Gousset

Julien Bondaz \& Julien Bonhomme

L'Offrande de la mort. Une rumeur au Sénégal Paris, CNRS Éd., 2017, 286 p., bibl., ill. («Bibliothèque de l'anthropologie»).

J uLien Bondaz et Julien Bonhomme analysent dans cet ouvrage la rumeur d'une offrande de la mort, qui a circulé au Sénégal en janvier 2010 et selon laquelle un mystérieux personnage, à bord d'une voiture $4 \mathrm{x} 4$, distribuerait à des mendiants une généreuse aumône composée de viande, d'un billet de 10000 francs CFa et d'un tissu en percale, ce qui provoquerait leur décès en même temps que l'enrichissement du donateur. À partir d'une ethnographie urbaine menée à Dakar, un an après les faits, les auteurs étudient cette 